\title{
Co-existing feedback loops generate tissue-specific circadian rhythms
}

\author{
J Patrick Pett ${ }^{1}$ (], Matthew Kondoff ${ }^{3}$, Grigory Bordyugov ${ }^{3}$, Achim Kramer $^{2}$ (D), Hanspeter Herzel ${ }^{3}$ (D)
}

Gene regulatory feedback loops generate autonomous circadian rhythms in mammalian tissues. The well-studied core clock network contains many negative and positive regulations. Multiple feedback loops have been discussed as primary rhythm generators but the design principles of the core clock and differences between tissues are still under debate. Here we use global optimization techniques to fit mathematical models to circadian gene expression profiles for different mammalian tissues. It turns out that for every investigated tissue multiple model parameter sets reproduce the experimental data. We extract for all model versions the most essential feedback loops and find auto-inhibitions of period and cryptochrome genes, Bmal1-Rev-erb- $\alpha$ loops, and repressilator motifs as possible rhythm generators. Interestingly, the essential feedback loops differ between tissues, pointing to specific design principles within the hierarchy of mammalian tissue clocks. Selfinhibitions of Per and Cry genes are characteristic for models of suprachiasmatic nucleus clocks, whereas in liver models many loops act in synergy and are connected by a repressilator motif. Tissue-specific use of a network of co-existing synergistic feedback loops could account for functional differences between organs.

DOI 10.26508/Isa.201800078 | Received 24 April 2018 | Revised 1 June 2018 | Accepted 4 June 2018 | Published online 14 June 2018

\section{Introduction}

Many organisms have evolved a circadian ( $24 \mathrm{~h}$ ) clock to adapt to the 24-h period of the day/night cycle (1). In mammals, physiological and behavioral processes show circadian regulation including sleep-wake cycles, cardiac function, renal function, digestion, and detoxification (2). In most tissues, about $10 \%$ of genes have circadian patterns of expression $(3,4)$. Surprisingly, the rhythmicity of clockcontrolled genes is highly tissue specific $(4,5,6)$.

Circadian rhythms are generated in a cell-autonomous manner by transcriptional/translational feedback loops (7) and can be monitored even in individual neurons (8) or fibroblasts (9).

Ukai and Ueda (10) depict the mammalian core clock as a network of 20 transcriptional regulators (10 activators and 10 inhibitors) acting via enhancer elements in their promoters such as E-boxes, D-boxes, and retinoic acid receptor-related orphan receptor elements (RRES). Because many of these regulators have similar phases of expression and DNA binding $(11,12)$, the complex gene regulatory network has been reduced by Korenčic et al (6) to just five regulators representing groups of genes: the activators Bmal1 and Dbp and the inhibitors Per2, Cry1, and Rev-Erba (Fig 1A and B).

Even this condensed network contains 17 regulations constituting multiple negative and positive feedback loops (13). To generate self-sustained oscillations, negative feedback loops are essential $(14,15)$. Originally, the self-inhibitions of the period and cryptochrome genes have been considered as the primary negative feedback loops (16). Later, computational modeling (17) and double-knockout experiments suggested that the Rev-Erb genes also play a dominant role in rhythm generation (18). Recently, it has also been shown that a combination of three inhibitors forming a repressilator (19) can reproduce expression patterns in the liver, adrenal gland, and kidney (13).

Despite many experimental and theoretical studies, major questions remain open: What are the most essential feedback loops in the core clock network? Do dominant loop structures vary across tissues?

Here, we use global optimization techniques to fit our five-gene model to expression profiles in different mammalian tissues (adrenal gland, kidney, liver, heart, skeletal muscle, lung, brown adipose, white adipose, suprachiasmatic nucleus (SCN), and cerebellum) (3). We find that for any given tissue, multiple parameter sets reproduce the data within the experimental uncertainties. By clamping genes and regulations at non-oscillatory levels (13), we unravel the underlying essential feedback loops in all these models. We find auto-inhibitions of the period and cryptochrome genes, Bmal1-Rev-erb- $\alpha$ loops, and repressor motifs as rhythm generators. The role of these loops varies between organs. For example, in the liver, repressilators dominate, whereas Bmal1-Rev-erb- $\alpha$ loops are found in the heart. Clustering of the model parameter sets reveals tissue-specific loop structures. For example, we rarely find the repressilator motif in the brain, heart, and muscle tissues because of the earlier phases and small amplitudes of cry1. We discuss that the co-existence of functional feedback loops increases robustness and flexibility of the circadian core clock.

\footnotetext{
${ }^{1}$ Institute for Theoretical Biology, Humboldt-Universität zu Berlin, Berlin, Germany ${ }^{2}$ Laboratory of Chronobiology, Charité-Universitätsmedizin Berlin, Berlin, Germany ${ }^{3}$ Institute for Theoretical Biology, Charité-Universitätsmedizin Berlin, Berlin, Germany
} 
A

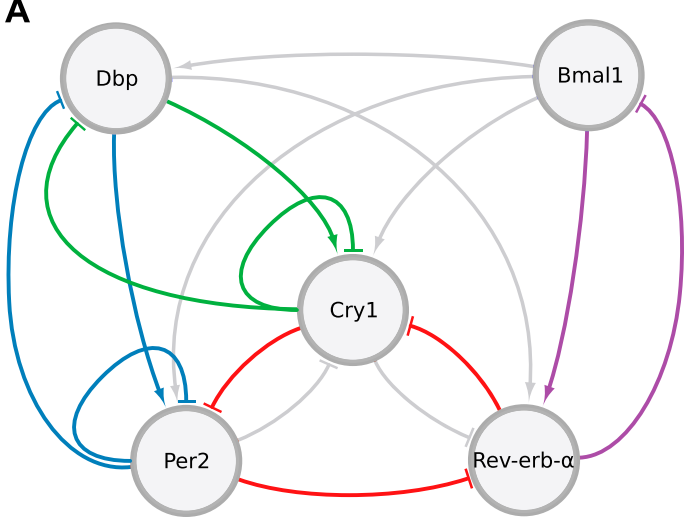

B

\begin{tabular}{cc}
\hline gene & represents \\
\hline Rev-erb- $\alpha$ & Rev-erb- $\alpha,-\beta$, Ror $\alpha,-\beta,-\gamma$ \\
$D b p$ & $D b p, H I f, T e f, E 4 b p 4$ \\
$B m a l 1$ & $B m a l 1$, Clock, Npas2 \\
Per2 & early E-box driven genes \\
Cry1 & late E-box driven gene \\
\hline
\end{tabular}

Figure 1. Network of the core clock model. (A) The graph comprises 7 activations and

10 inhibitions forming several negative feedback loops. Four loops that are mainly discussed in the literature and were most often found by our analysis are marked in different colors. Note that for Per2 and Cry1 autoinhibitions also, extensions via the gene Dbp are counted. (B) Table of genes represented by each variable of the model.

\section{Results}

\section{A five-gene regulatory network represents most essential loops}

Here, we derive a gene regulatory network that can be fitted successfully to available transcriptome, proteome, and ChIP-seq data. We will use the model to explore tissue-specific regulations.

Many circadian gene expression profiles for mouse tissues are available $(5,20,21)$. Here, we focus on data sets from tissues spanning $48 \mathrm{~h}$ with a 2 -h sampling (3). These comprehensive expression profiles are particularly well suited to study tissue differences. For mouse liver also, proteome (22) and ChIP-seq data $(11,23,24)$ are available with lower resolution.

Using global parameter optimization, we fit tissue-specific model parameters directly to the gene expression profiles of Zhang et al (3). Proteome and ChIP-seq data are primarily used to specify reasonable ranges of the delays between transcription and the action of activators and repressors. The ranges of degradation rates have been adapted to large-scale studies measuring half-lifes of mRNAs $(25,26)$ and proteins $(27)$.

Quantitative details of activation and inhibition kinetics are not known because of the high complexity of transcriptional regulation. The transcriptional regulators are parts of MDa complexes (28) including histone acetyltransferases and histone deacetylases (29). Details of DNA binding, recruitment of co-regulators, and histone modifications are not available (30). Thus, we use heuristic expressions from biophysics (31) to model activation and inhibition kinetics. Exponents represent the number of experimentally verified binding sites (32) (Supplementary Information 1), and the parameters were assumed to be in the range of the working points of regulation.

To justify the topology of our reduced gene regulatory network, we analyze the amplitudes and activation phases of all the 20 regulators described in Ukai and Ueda (10) (Fig 2). Repressor phases were inverted by $12 \mathrm{~h}$ to reflect the maximal activity and allowing direct comparison with activators.

Fig 2 shows that the five genes binding to RREs and the four genes binding to D-boxes cluster at specific phases. Consequently, we represent these regulators by selected genes with large amplitudes: Rev-erb- $\alpha$ and Dbp. Because the other RRE and D-box regulators peak at similar or directly opposed phases, their additional regulation can be taken into account by the fitting of activation and inhibition parameters.
The regulation via E-boxes is quite complex $(11,30,33,34)$. In addition to the activators Bmal1 and Bmal2, we have their dimerization partners Clock and Npas2 and their competitors Dec1 and Dec2. Furthermore, there are the early E-box targets Per1, Per2, Per3, and Cry 2 and the late gene Cry1. We model this complicated modulation by three representative genes: Bmal1 as the main activator and Per2 and Cry1 as the early and late E-box target, respectively.

In summary, the reduced gene regulatory network consists of five genes and 17 regulations (Fig 1). All regulations and the number of binding sites have been confirmed by several experimental studies discussed in detail in (32). Interestingly, liver proteomics (22) and ChIP-seq data are consistent with morning activation via Bmal1, evening activation by Dbp, and sequential inhibition by Rev-erb- $\alpha$, Per2, and Cry1. Recent detailed biochemical experiments support the notion that there are distinct inhibition mechanisms associated with Per2 and Cry1 (30). The essential role of the late Cry1 inhibition has been stressed also by Ukai-Tadenuma et al (35) and Edwards et al (36).

As discussed above, our model is fitted directly to mRNA time series collected for different tissues at 2-h intervals for a total duration of $2 \mathrm{~d}$. The transcriptional/translational loops are closed by delayed activation or repression realized by the corresponding proteins. Because most quantitative details of posttranscriptional modifications, complex formations, nuclear import, and epigenetic regulations are not known, we simplify all these intermediate processes by using explicit delays. Thus, we describe the core clock network by five delay-differential equations with 34 kinetic parameters (see Supplementary Information 1 for the complete set of equations).

The model constitutes a strongly reduced network that approximates the highly complex protein dynamics by delays. Inhibition strengths are represented by a single parameter, whereas modeling of activation requires two parameters: maximum activation and threshold levels. While keeping in mind the proposed simplifications, the resulting tissue-specific models can still be regarded as a biologically plausible regression of the underlying biological dynamics.

In Fig 3A, we show examples of simulations fitted to the corresponding gene expression patterns. After successful parameter optimization as described below, the differences between data and models are comparable with experimental uncertainties quantified by comparing different studies $(3,21,37)$ and by studying the 


\section{A Histogram of phases (all tissues)}

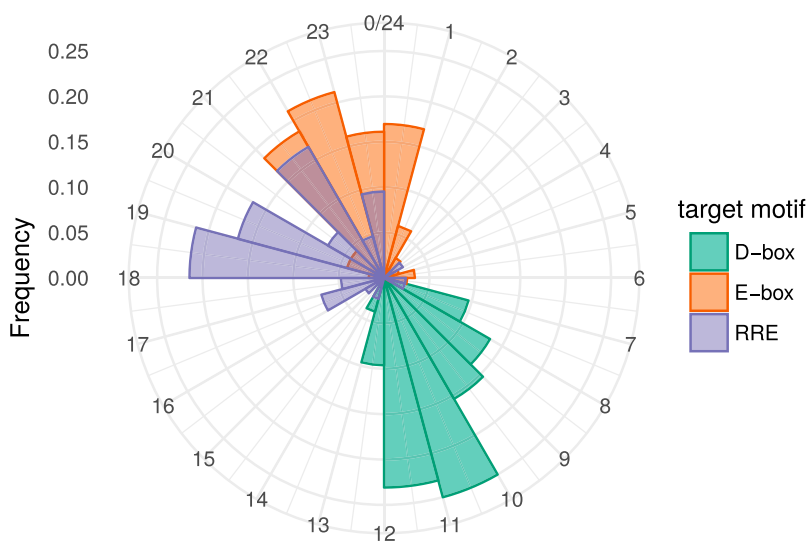

B

\begin{tabular}{|l|}
\hline Bmal1 \\
Bmal2 \\
Clock \\
Npas2 \\
Cry1 \\
Cry2 \\
Dec1 \\
Dec2 \\
Per1 \\
Per2 \\
Per3 \\
\hline
\end{tabular}

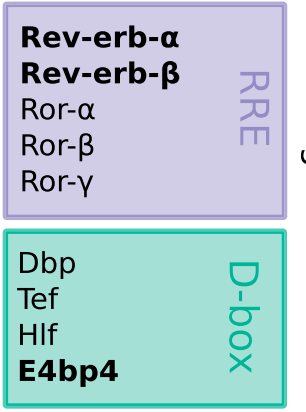

C

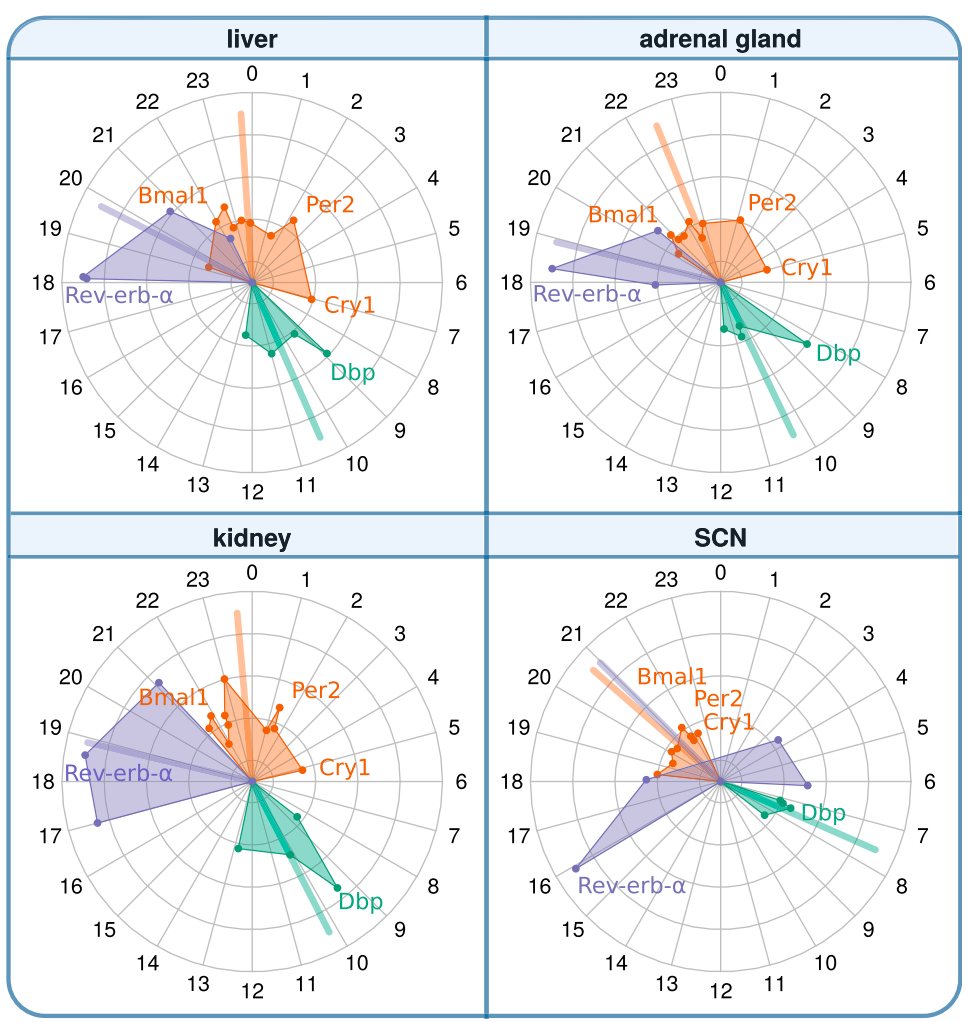

Figure 2. Circular plots of 20 regulators reveal redundancies and serial inhibition $(10,12)$.

They represent peak phases of mRNA expression in multiple tissues (3). Note that repressor phases were inverted by $12 \mathrm{~h}$ to allow direct comparison with activators. (A) Histogram of the phase distribution over all tissues. (B) List of genes represented in circular plots and their corresponding target motifs. Repressors are marked in bold. (C) Phases of core clock genes in selected tissues. Colored lines correspond to the circular mean of the respective groups. Amplitudes are linearly scaled. The differences between SCN and other tissues are particularly notable (e.g., the earlier Cry1 peak). Antagonistic regulations of Rev-erb and Ror in the SCN can be modeled by reduced inhibition strength of Rev-erb- $\alpha$.

differences between the first and second day of the expression profiles by Zhang et al (3) (Supplementary Information 3).

\section{Vector field optimization (VFO) improves model fitting}

To investigate whether our reduced gene regulatory network can reproduce tissue-specific data, (3) we developed a pipeline for global parameter optimization and analysis (scheme in Fig 3B). We applied the pipeline multiple times to each tissue-specific expression profile, allowing us to compare optimized model parameters between tissues. Table 1 lists the number of optimization runs for 10 analyzed tissues. Four tissues (liver, SCN, adrenal gland, and kidney) are discussed in more detail in the following sections, whereas results for others can be found in Supplementary Information 2 and 5 .

The agreement of model simulations and experimental mRNA time courses (3) were measured by a scoring function. In this

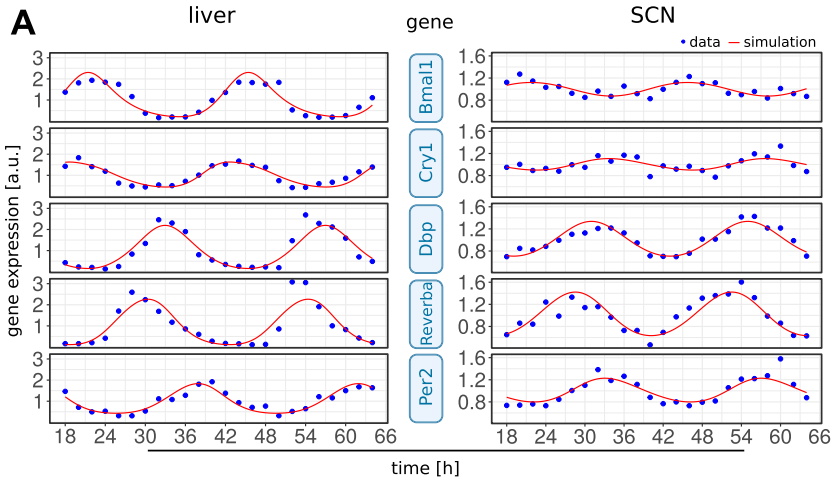

B

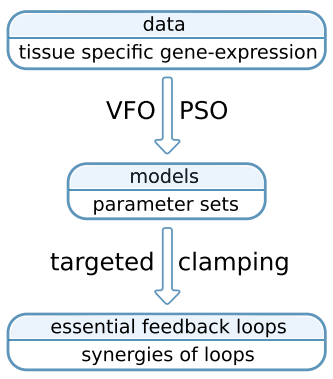

Figure 3. (A) Example time series for data and simulation. One fit in liver (left) and one fit in the SCN (right) are shown. Expression levels are normalized to the mean values. The liver fit has a score of 0.01 and involves the Bmal1-Rev-erb- $\alpha$, repressilator, and Cry1 loops, whereas the SCN fit scores 3.36 and involves the Bmal1-Rev-erb- $\alpha$, Per2, and Cry1 loops. Note the smaller amplitudes and the early Cry1 phase in the SCN. (B) Workflow of the analysis. Multiple optimized parameter sets are obtained from each tissue-specific data set. Then essential loops are identified in the respective models. 
Table 1. Number of optimization runs per tissue and average score.

\begin{tabular}{lllllll|llll} 
& Adrenal gland & Kidney & Liver & Heart & Skeletal muscle & Lung & Brown adipose & White adipose & SCN & Cerebellum \\
\hline Number of runs & 100 & 93 & 57 & 57 & 58 & 31 & 62 & 45 & 153 & 58 \\
\hline Runs with score $<10$ & 66 & 59 & 52 & 44 & 35 & 21 & 36 & 22 & 46 & 39 \\
\hline Mean score & 3.84 & 4.48 & 1.58 & 3.74 & 5.17 & 3.04 & 4.00 & 3.24 & 7.21 & 3.99 \\
\hline Figure & $2,5,6,7,8$ & $2,5,6,7,8$ & $2-8$ & 8, S2, S5 & 8, S2, S5 & 8, S2, S5 & 8, S2, S5 & 8, S2, S5 & 2-8 & S2, S5 \\
\hline
\end{tabular}

Four tissues (adrenal gland, kidney, liver, and SCN) are mainly discussed in the main text and others are described in supplements as indicated in the last row.

function period, relative phases and fold changes of measured gene transcripts are taken into account. The complete scoring function is given in Supplementary Information 3.

Model parameters are chosen by global optimization, such that the score obtained by our scoring function is minimal. The optimization method approaches a local minimum in a high dimensional parameter space, and thus, final scores of each run depend on the starting conditions. We only used model fits with scores lower than a chosen threshold of 10 for further analyses. A cutoff of 10 reflects deviations that are within the experimental uncertainties according to our tolerance values (Supplementary Information 3). Interestingly, the fractions of optimization runs with scores lower than 10 vary across tissues. Whereas the largest number of successful runs is found for liver data (about 90\%), for the kidney and adrenal gland about $2 / 3$ and for SCN only $1 / 3$ of the runs yield good scores below the chosen threshold.

Allowed ranges for parameters were defined to restrict the search space. Although delays and degradation rates are optimized within biologically plausible ranges around experimentally measured values, for activation and inhibition strengths no such measurements are available. Therefore, we define ranges based on oscillation mean levels and corresponding to the working points of regulations, that is, ranges in parameter space in which regulation strengths vary most.

Global optimization is performed with particle swarm optimization (PSO) (38). A number of particles-each representing one parameter combination-are initialized randomly using Latin hypercube sampling (39) and moved around in the parameter space with velocities changing according to both their individual and their neighbor's known best location. The movements are conducted for a number of iterations while velocities decrease and particles converge to an optimum.

We improve global optimization by identifying good starting conditions. To this end, we devise a strategy which we here call "vector field optimization." Our algorithm makes use of experimentally-derived time courses for model variables and their mathematical description in terms of differential equations. From the data, we can approximate the time derivatives together with the right-hand sides of our model equations (Fig $4 \mathrm{~A}$ and Supplementary Information 4). By minimizing the differences, we obtain initial values of model parameters. This step does not require simulation, but already yields parameter combinations that account for much of the differences between time courses. For example, the known antiphase oscillations of Bmal1 and Rev-erb- $\alpha$ can be generated with a Bmal1 delay of about $6 \mathrm{~h}$. Even though the overall search space of this parameter is the interval from 0 to $6 \mathrm{~h}$,
VFO leads to initial delay values close to $6 \mathrm{~h}$ (see Supplementary Information 4 for details).

VFO is performed using a bounded gradient method to ensure that solutions lie within the parameter limits. Starting points for the gradient method are chosen randomly. We tested whether VFO improves the scores of model fits. Indeed, we are able to find significantly more good fits for the liver and SCN than with PSO alone (Fig 4B). Notably, in the SCN, it was difficult to reach scores lower than 10 without previous application of VFO.

\section{Clamping reveals essential loops}

Using global optimization, we found for all 10 tissue-specific expression profile (3) parameter sets that reproduce the data within experimental uncertainties (Supplementary Information 2 and 3).

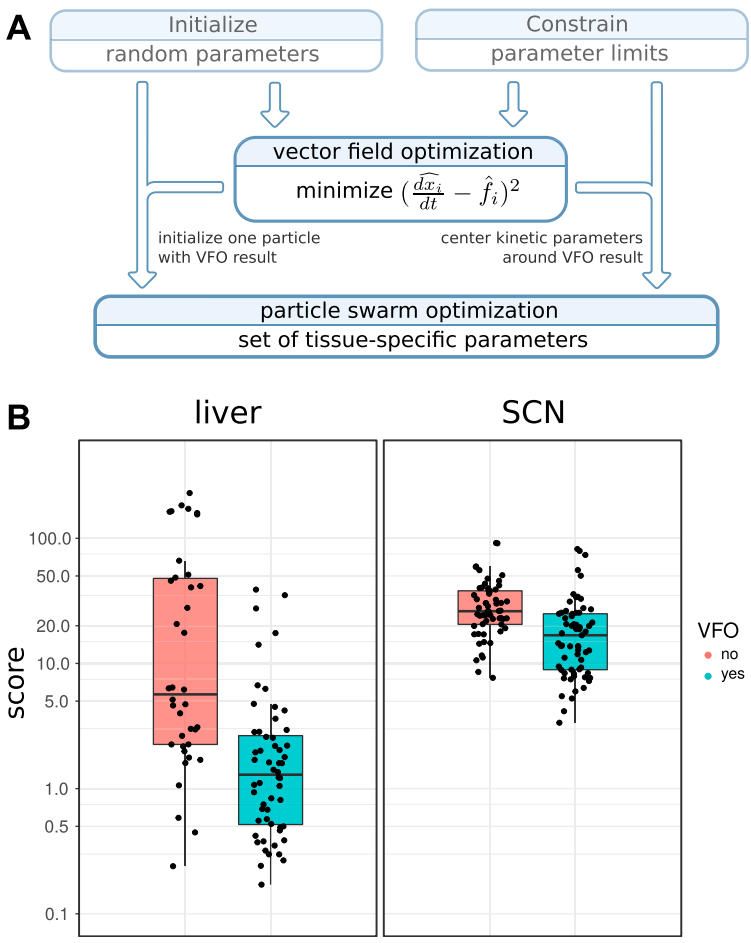

Figure 4. Improved fitting with VFO.

(A) Flow diagram showing how VFO is integrated into the fitting procedure. The resulting parameter set is used to initialize one particle and to pre-emphasize kinetic parameters. (B) Score for fits to circadian transcription data from mouse liver and SCN with and without VFO. Each point represents a fitted model. VFO leads to significantly lower score values (Wilcoxon rank-sum test, $P$-value liver: $4.29 \times 10^{-7}$, P-value SCN: $4.819 \times 10^{-6}$ ). 
There was not just a single optima of global optimization, but for all investigated tissues, multiple parameter configurations fitted the data.

To determine essential feedback loops for each model fit, we use our clamping protocol published in 2016 (13). Clamping of genes is carried out by setting the expression level of genes to their mean value (constant) and corresponds to constitutive expression experiments in the wet laboratory $(36,40,41,42)$. It allows comparison of the effect of rhythmic versus basal regulation.

In addition to gene clamping, we also clamp specific regulations via gene products. In silico, this is carried out by setting the corresponding terms in the differential equations of the model constant. Regulations/terms are shown as network links in Fig 1. We can examine the relevance of feedback loops associated with such links by clamping regulations systematically.

To reduce computational effort, we use a targeted clamping strategy, testing specifically which feedback loops are essential. We regard a negative feedback loop as essential for oscillations if clamping of each link that is part of the loop disrupts rhythmicity (only one link at a time is clamped). Details are provided in Supplementary Information 5.

In addition, we test the synergy of loops by clamping combinations of regulations. We are able to distinguish two different modes of synergistic function: (i) two loops work independent of each other and mutually compensate for perturbations, and (ii) two dependent loops share the required feedback for oscillations, such that rhythms only occur when both loops are active.

For example, in the liver, we found many model fits in which the Bmal1-Rev-erb- $\alpha$ and repressilator loops function synergistically. Both loops constitute a negative feedback from Rev-erb- $\alpha$ onto
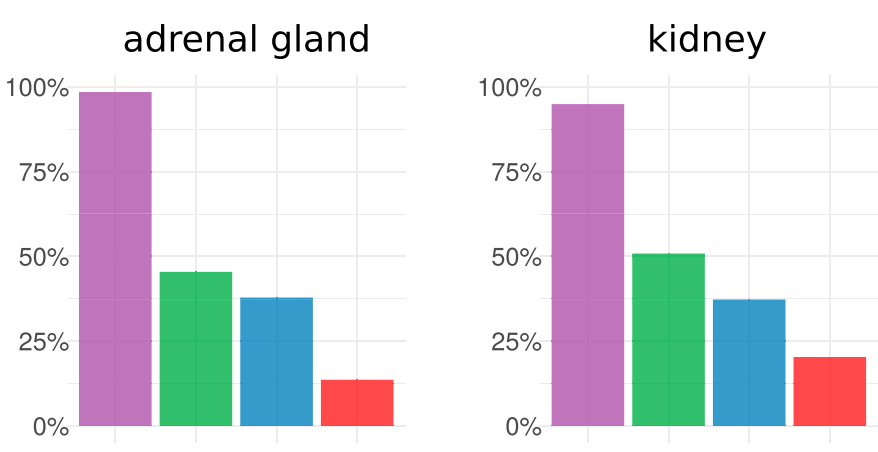

Loops:

Bmal1/Rev-erb- $\alpha$

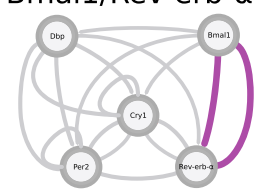
across tissues.

For each tissue-specific transcriptome data set multiple model fits were generated. In each model fit, essential feedback loops were then identified using clamping analysis. Frequencies are shown for a set of four core loops that were most prominent in the analysis and are discussed in the literature.
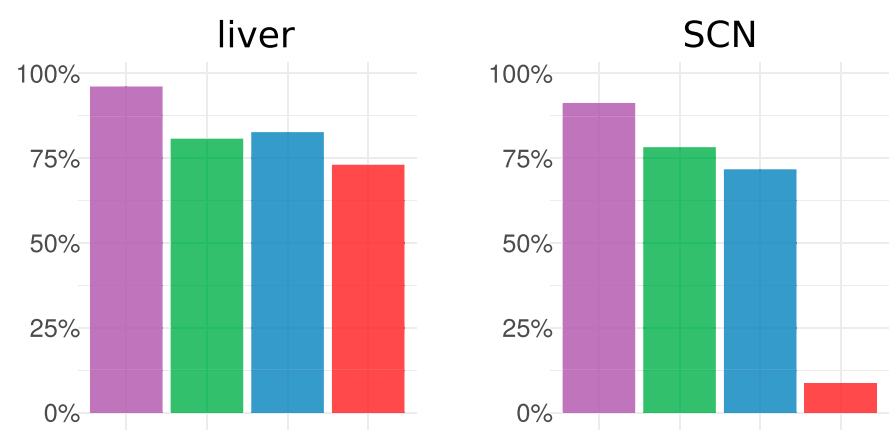

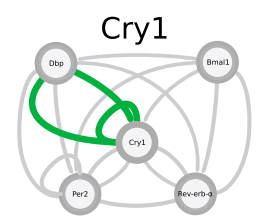

Per2

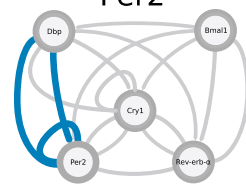

repressilator

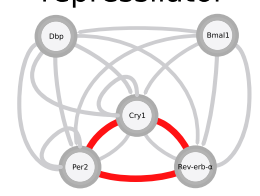


To find out whether tissue differences are reflected in the model parameters, we examine their distributions in the 34-dimensional parameter space. To this end, we perform dimensionality reduction by principal component analysis and visualize tissue differences using linear discriminant analysis (45).

Fig $6 \mathrm{~A}$ illustrates that the parameters sets fitted to SCN are clearly different from those fitted to other tissues. The differences can be assigned to selected parameters as indicated by the red arrows.

In Fig 6B, we project the model parameters to the first two principal components and color the points according to the essential loops. It turns out that Per2 loops (blue), Cry1 loops (green), and Bmal1-Rev-erb- $\alpha$ loops (orange) are associated with distinct parameter sets. We observe, for example, an association of Cry1 loops with high Cry1 delay.

The differences in loop distributions (Fig 5) and parameter constellations (Fig 6) suggest that differences between expression profiles (Fig 2) imply tissue-specific mechanisms to generate selfsustained oscillations. For example, in brain tissues, small amplitudes and early Cry1 phases promote self-inhibitions of Per2 and Cry1, whereas a large Rev-erb- $\alpha$ amplitude in liver leads to many solutions with Bmal1-Rev-erb- $\alpha$ loops and repressilators.

\section{Synergies of feedback loops}

So far, we discussed tissue-specific frequencies of single loops. Interestingly, most parameter sets cannot be assigned to unique loops but to combinations of different essential feedback loops. Now, we use a targeted clamping strategy (Supplementary Information 5) to explore possible synergies of feedback loops.

Our clamping strategy allows us to find loops that are necessary (or essential) for rhythm generation. If we clamp regulations that are part of these loops, rhythms vanish. Furthermore, by clamping many regulations at the same time, we can also identify sets of loops that are sufficient for oscillation generation. In simulations, rhythms persist if these loops are active while all others are clamped. We term such synergistic sets of loops "rhythm-generating oscillators."

Analyzing 420 parameter sets, we find more than $70 \%$ that exhibit synergies of different feedback loops. Fig 7A illustrates that most models constitute combinations of loops. Venn diagrams in Fig 7B show that in liver, Bmal1-Rev-erb- $\alpha$ loops together with repressilators form the largest group of oscillators, whereas in the SCN, Bmal1-Reverb- $\alpha$ loops are typically associated with Per2 and Cry1 loops.

Interestingly, the synergy of multiple loops leads typically to low scores. There is a significant difference in the number of loops between parameter sets greater than and lower than the median score (Wilcoxon rank-sum test, $P$-value $<0.0001$ ) and most model fits involving all four loops lead to excellent scores lower than 2.5.

Moreover, repressilators exhibit quite good scores, in particular, for the liver, kidney, and adrenal gland. For these tissues, fits with repressilator have an average score of 1.24, whereas fits without repressilator have a mean score of 4.41. This is consistent with our finding that better scores involve more loops. The repressilator motif connects inhibitions of Per2, Cry1, and Rev-erb- $\alpha$ and links the loops synergistically.

\section{Discussion}

Circadian rhythms in mammals are generated by a cellautonomous gene regulatory network (46). About 20 regulators drive core clock genes via E-boxes, D-boxes, and RREs (10). Based on clustered gene expression phases (compare Fig 2), we reduced the system to a network of five genes connected by 7 positive and
A

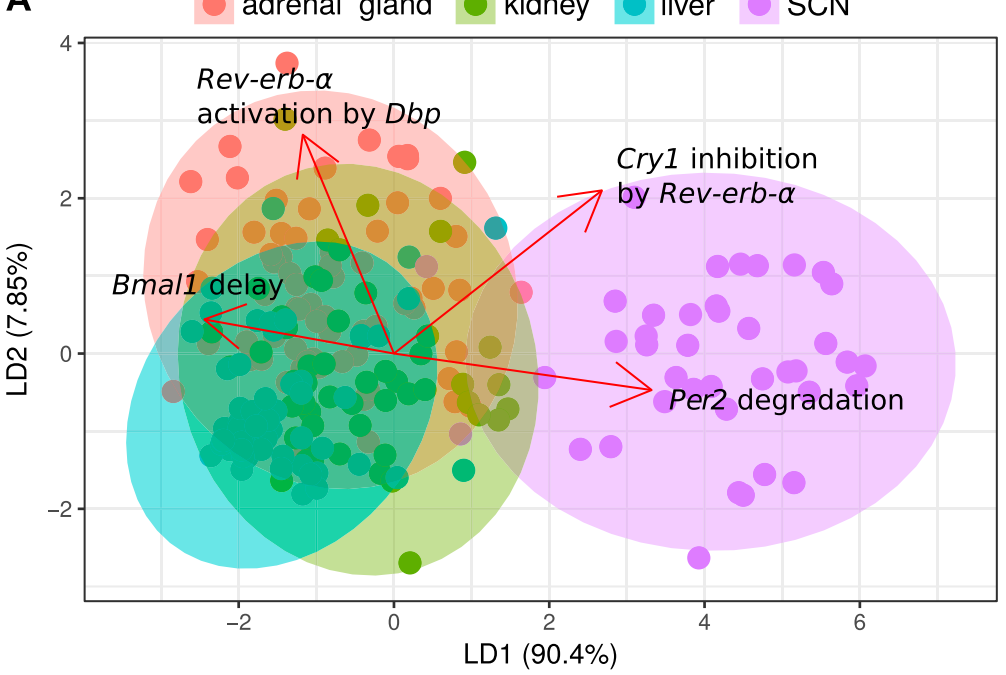

B $\quad$ Loop: $\backsim$ Cry $1 \bullet$ Per2 $\bullet$ Repr $\square$ Rev-erb- $\alpha$

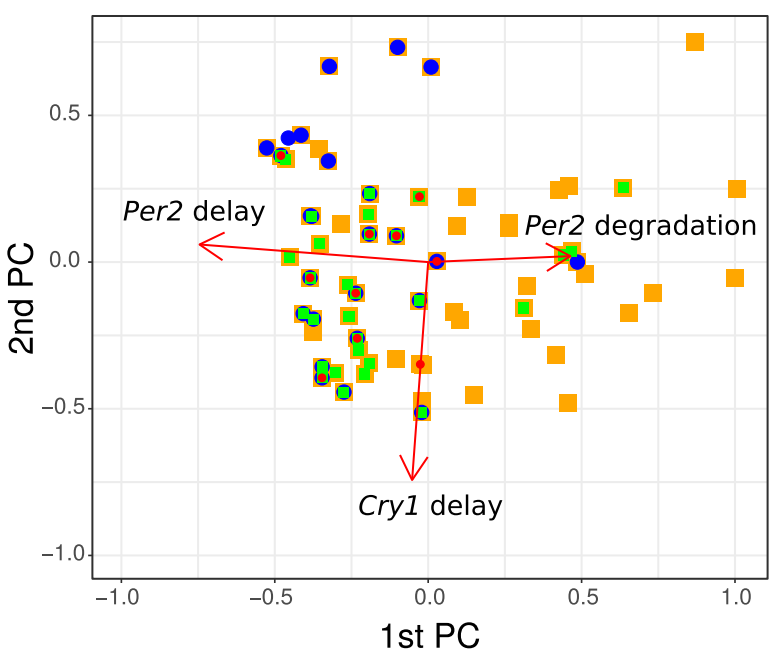

Figure 6. Tissue-specific models separated in parameter space.

(A) Linear discriminant analysis. Fits (points) are projected to a plane while trying to maximize the variance between tissues. The projected parameter vectors are visualized as arrows in this plane, showing how parameters differ between tissues. Only the four largest arrows are shown for simplicity. (B) Loops in parameter space. Shown are the first two principal components and points corresponding to parameter sets for the adrenal gland. Directions of the parameter axes are given as red arrows. Relations between parameter values and loops are visible, for example essential Cry1 loops (green) occur, when Cry1 delays are large. Only the three largest arrows are shown, which are markedly larger than the others. 
A

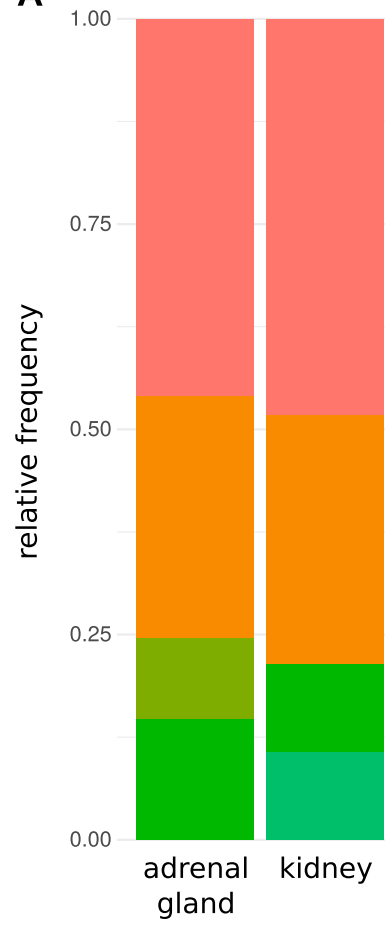

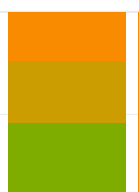

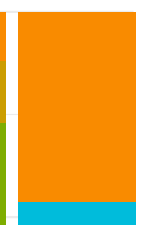

Loops
Bmal1/Rev-erb- $\alpha$
Bmal1/Rev-erb- $\alpha+$ Cry 1
Bmal1/Rev-erb- $\alpha+$ Cry $1+$ Cry $1 /$ Dbp

+ Repressilator

Bmal1/Rev-erb- $\alpha+$ Cry $1+$ Repressilator

Bmal1/Rev-erb- $\alpha+$ Per2

Bmal1/Rev-erb- $\alpha+$ Per2/Dbp

Bmal1/Rev-erb- $\alpha+$ Per2/Dbp + Cry1

Bmal1/Rev-erb- $\alpha+$ Per2/Dbp + Cry1

+ Cry $1 / \mathrm{Dbp}$

Bmal1/Rev-erb- $\alpha+$ Per2/Dbp + Cry1

+ Repressilator

Bmal1/Rev-erb- $\alpha+$ Per2/Dbp

+ Repressilator

Bmal1/Rev-erb- $\alpha+$ Per2 + Per2/Dbp

Bmal1/Rev-erb- $\alpha+$ Per2 + Per2/Dbp

+ Cry1/Dbp

Per2/Dbp + Cry1 + Repressilator
B

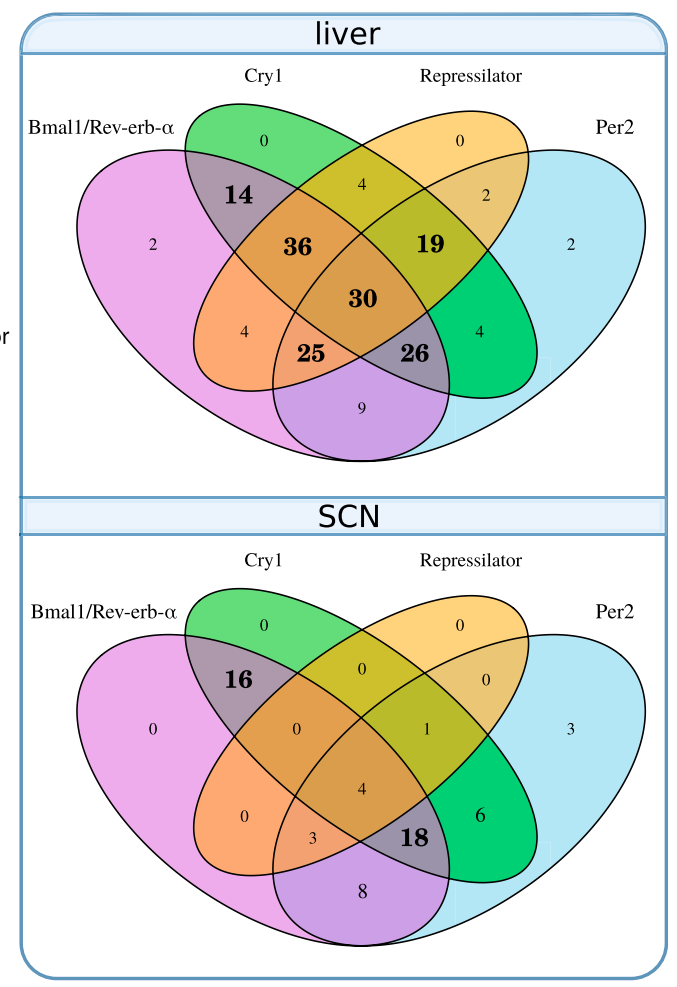

Figure 7. Proportions of minimal oscillators across tissues.

(A) Frequencies of oscillators in models of different tissues. An oscillator comprises one or more loops (connected with a "+" in the legend). Per2, Cry1 loops, and their extensions via Dbp (Fig 1) are counted separately here. (B) Venn diagrams of oscillator composition for liver and SCN. Bold numbers highlight the most frequent subsets. In liver, most oscillators comprise many loops including Bmal1-Rev-erb- $\alpha$ loop, repressilator, Per2, and Cry1 loops. In the SCN most oscillators are a combination of Bmal1-Rev-erb- $\alpha$ with Cry1 and Per2.

10 negative regulations (Fig 1). This reduced model still contains multiple positive and negative feedback loops.

Our aim was to identify the most essential feedback loops and to quantify tissue differences. Our network model was fitted to comprehensive expression profiles of 10 mammalian tissues (3). Furthermore, we use proteomics data (22), ChIP-seq data (11, 23), and decay-rate data (25) to constrain the ranges of unknown parameters. Since quantitative data on protein dynamics are sparse, we simplified the model by using explicit delays between transcription and regulation.

We optimized parameters by a combination of a novel approach termed VFO and PSO (38). After combining these global optimization techniques, our simulations could reproduce the data within experimental uncertainties.

To our surprise, we found for every studied tissue multiple excellent fits with quite different parameter constellations. To extract the responsible feedback loops we performed a systematic clamping analysis. Individual regulatory terms (edges in the network) were systematically clamped to constant values. These clamping methods revealed the essential feedback loops in each of the networks derived from tissue-specific expression profiles.

We found an astonishing diversity of essential feedback loops in models that were able to reproduce the experimental data. Among the essential loop structures we found Per and Cry selfinhibitions. These loops have been considered as the primary negative feedacks because the double knockouts of Cry genes (47) and the triple knockouts of Per genes (48) were arrhythmic. Later, additional feedback loops via nuclear receptors have been found (49). As predicted by modeling (17) and confirmed by Rev-erb double knockouts (18), the Bmal1/Rev-erb loops constitute another possible rhythm generator. Indeed, in all tissues, we detected parameter constellations that use this negative feedback loop.

Recently, the repressilator, a chain of serial inhibitions, was suggested as a possible mechanism to generate oscillations in the liver and adrenal gland (13). This loop structure is associated with dual modes of E-box inhibitions (30) based late Cry1 expression (35) and late CRY1 binding to E-boxes (11). Because the expression phase of Cry1 is tissue-dependent, it is plausible that the detection of repressilators also might differ between different organs.

Indeed, repressilators are less frequently detected as essential in models based on brain data-derived parameter sets as shown in Fig 5. In general, the model parameters in the SCN are clearly different from the parameters in peripheral tissues (Fig 6). Thus, modeling can point to different design principles in specific organs. The large amplitudes and late Cry1 phases in tissues such as liver suggest that the repressilator is a relevant mechanism in these tissues, whereas small amplitudes and early cry1 phase in brain tissues favor Cry and Per self-inhibitions.

Major differences between tissues have been reported also regarding amplitudes and phases of clock-controlled genes $(3,4,5,6)$. Such differences are presumably induced by tissue-specific 
transcription factors $(34,50,51)$. Moreover, different organs receive different metabolic and neuroendocrine inputs leading to quite different rhythmic transcriptomes. These systemic tissue differences can also modify the core clock dynamics. In particular, nuclear receptor rhythms differ drastically between organs (52) and can induce tissue specificities of the core clocks $(53,54)$.

Interestingly, the best scoring models involve several essential feedback loops. This observation indicates that the synergy of different feedback mechanisms improves oscillator quality. Furthermore, co-existing loops imply redundancy, and thus, the core clock is buffered with respect to non-optimal gene expression, hormonal rhythms, seasonal variations, and environmental fluctuations.

Co-occuring feedback regulations might also explain reports where different circadian outputs displayed slightly different periods. For example, in SCN slices, different reporter signals indicated distinct periods $(55,56)$, and also in crickets, two independent negative feedback loops were reported (57). In some of our highscoring networks, we indeed find two independent frequencies leading to slight modulations of the circadian waveforms (Supplementary Information 6).

Tissue-specific core clock mechanisms are presumably related to functional differences of SCN and peripheral organs. Per gene regulations are particularly important in the SCN because light inputs and coupling via vasoactive intestinal peptide induce Per genes via cAMP response element-binding protein $(16,58)$. Relatively small core clock amplitudes in the SCN allow efficient entrainment and synchronization $(59,60)$. Moreover, small amplitudes might facilitate adaptation to long and short photoperiods by varying coupling mechanisms $(61,62)$. The dominant role of Per and Cry self-inhibitions is also reflected by the arrhythmic activities of Per and Cry double knockouts $(47,48)$.

Peripheral organs such as the liver, kidney, and adrenal gland govern the daily hormonal and metabolic rhythms. Consequently, large amplitudes and pronounced rhythms of nuclear receptors are observed $(52,63)$. Interestingly, we find that feedback loops involving RREs are more prominent in these tissues (compare Fig 8).

We now address the question of how the choice of our simplified model might affect the results. As shown in Table 1, we use many different parameter sets that can reproduce the data within experimental uncertainties. Such a probabilistic interpretation makes our conclusions more robust regarding the choice of parameters. Furthermore, a fit to different qPCR data sets (6) also detected the repressilator motif as a core element in the liver and adrenal gland. The model structure was chosen to be generic and, in particular, not depending on specific molecular mechanisms. It was created in an unbiased way using general assumptions and experimental evidence on interactions. Interestingly, the co-existence of Per/Cry and Bmal1/Rev-erb- $\alpha$ loops has been found also in a different larger model (17). Thus, we assume that the main results of our study do not depend much on the model choices made.

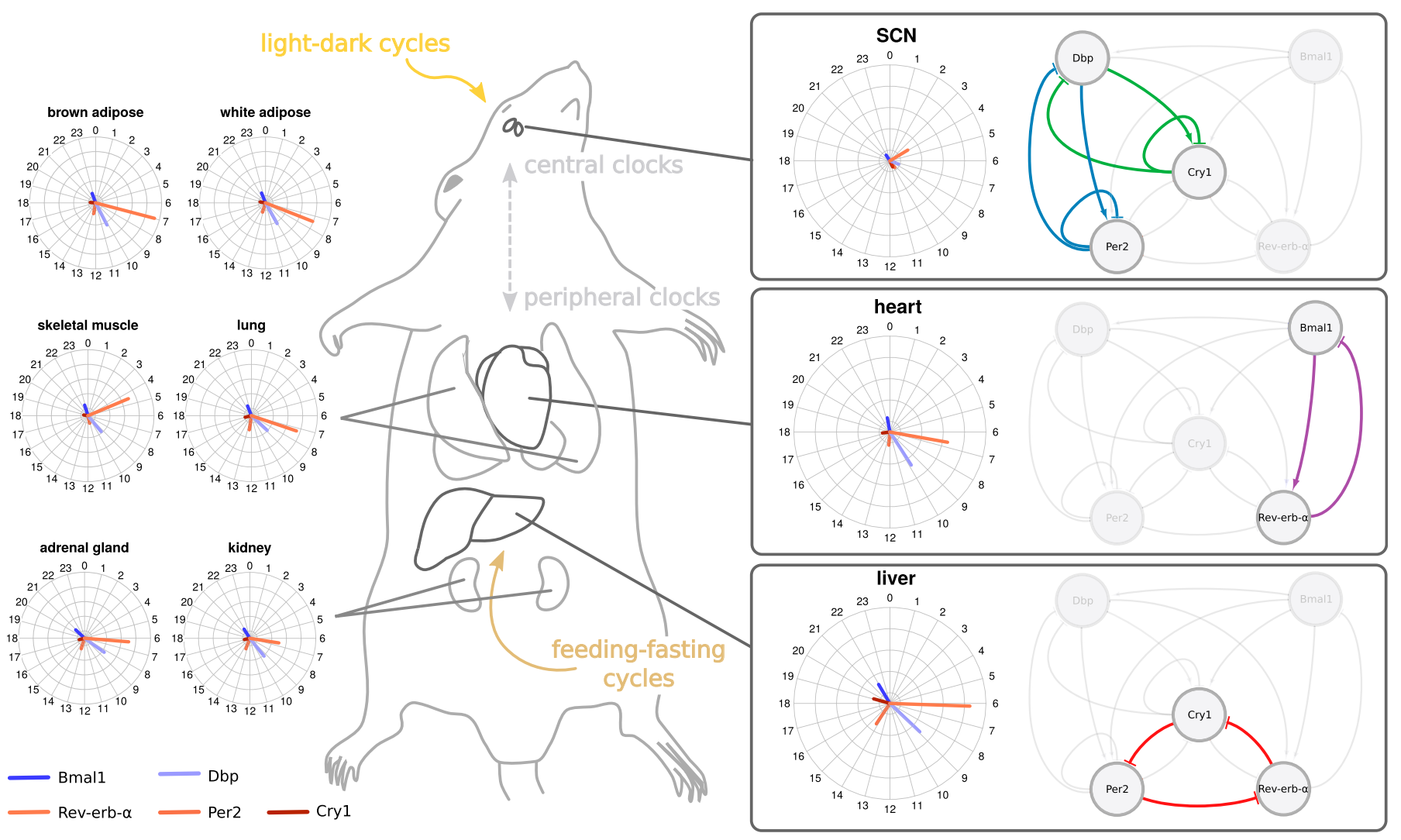

Figure 8. Differences between gene expression in mouse tissues and associated feedback loops found in model fits Shown are three representative feedback loops for tissues as characteristic examples. 
To test our predictions, we suggest tissue-specific modifications of the core clock genes. In particular, constitutive or out-of-phase expression of clock genes can be used. It has been shown already that constitutive expression of Per and Cry genes impairs rhythms $(36,40,41)$. Also, specific regulations can be manipulated experimentally to resemble clamping of regulatory edges. For example, the removal of intronic RREs of the Cry1 gene leads to vanishing amplitudes in single cells (35). Moreover, available REV-ERB agonists (63) could be applied to study the role of the corresponding loops. Therefore, our model predictions can be tested by specific perturbations resembling our numerical interventions.

In summary, our study suggests that there is not necessarily a single dominant feedback loop in the mammalian core clock. Instead, multiple mechanisms including Per/Cry self-inhibitions, Bmal1/Rev-erb loops, and repressilators are capable to generate circadian rhythms. The co-existence of feedback loops provides redundancy and can thus enhance robustness and flexibility of the intertwined circadian regulatory system.

\section{Materials and Methods}

Methods can be found in the Supplementary Information.

\section{Supplementary Information}

Supplementary Information is available at https://doi.org/10.26508/lsa. 201800078.

\section{Acknowledgements}

We are grateful for discussions with Dr. Christoph Schmal, Dr. Bharath Ananthasubramaniam, Dr. Anja Korenčič, and Dr. Matthias König. This work was supportet by grants from Deutsche Forschungsgemeinschaft (HE2168/ 11-1 and TRR/SFB 186 A16 and A17), Bundesministerium für Bildung und Forschung (01GQ1503), and Graduiertenkolleg CSB 1772/2.

\section{Author Contributions}

JP Pett: conceptualization, data curation, software, formal analysis, validation, investigation, visualization, methodology, and writing-original draft, review, and editing.

M Kondoff: conceptualization, methodology, and writing-review and editing.

G Bordyugov: conceptualization, supervision, and writing-review and editing.

A Kramer: supervision, funding acquisition, and writing-review and editing.

H Herzel: conceptualization, supervision, funding acquisition, writing-original draft, project administration, and writing-review and editing.

\section{Conflict of Interest Statement}

The authors declare that they have no conflict of interest.

\section{References}

1. Dunlap JC (1999) Molecular bases for circadian clocks. Cell 96: 271-290 doi:10.1016/s0092-8674(00)80566-8

2. Kramer A, Merrow M (2013) Circadian Clocks. Berlin/Heidelberg, Germany: Springer.

3. Zhang R, Lahens NF, Ballance HI, Hughes ME, Hogenesch JB (2014) A circadian gene expression atlas in mammals: Implications for biology and medicine. Proc Natl Acad Sci USA 111: 16219-16224. doi:10.1073/ pnas. 1408886111

4. Mure LS, Le HD, Benegiamo G, Chang MW, Rios L, Jillani N, Ngotho M, Kariuki T, Dkhissi-Benyahya O, Cooper HM, et al (2018) Diurnal transcriptome atlas of a primate across major neural and peripheral tissues. Science 359: eaao0318. doi:10.1126/science.aao0318

5. Storch KF, Lipan O, Leykin I, Viswanathan N, Davis FC, Wong WH, Weitz CJ (2002) Extensive and divergent circadian gene expression in liver and heart. Nature 417: 78-83. doi:10.1038/nature744

6. Korenčič A, Košir R, Bordyugov G, Lehmann R, Rozman D, Herzel H (2014) Timing of circadian genes in mammalian tissues. Sci Rep 4: 5782. doi:10.1038/srep05782

7. Hardin PE, Hall JC, Rosbash M (1990) Feedback of the Drosophila period gene product on circadian cycling of its messenger RNA levels. Nature 343: 536-540. doi:10.1038/343536a0

8. Welsh DK, Logothetis DE, Meister M, Reppert SM (1995) Individual neurons dissociated from rat suprachiasmatic nucleus express independently phased circadian firing rhythms. Neuron 14: 697-706. doi:10.1016/0896-6273(95)90214-7

9. Nagoshi E, Saini C, Bauer C, Laroche T, Naef F, Schibler U (2004) Circadian gene expression in individual fibroblasts: Cell-autonomous and selfsustained oscillators pass time to daughter cells. Cell 119: 693-705. doi:10.1016/j.cell.2004.11.015

10. Ukai H, Ueda HR (2010) Systems biology of mammalian circadian clocks Annu Rev Physiol 72: 579-603. doi:10.1146/annurev-physiol-073109-130051

11. Koike N, Yoo SH, Huang HC, Kumar V, Lee C, Kim TK, Takahashi JS (2012) Transcriptional architecture and chromatin landscape of the core circadian clock in mammals. Science 338: 349-354. doi:10.1126/ science.1226339

12. Westermark PO, Herzel H (2013) Mechanism for $12 \mathrm{hr}$ rhythm generation by the circadian clock. Cell Rep 3: 1228-1238. doi:10.1016/j.celrep.2013.03.013

13. Pett JP, Korenčič A, Wesener F, Kramer A, Herzel H (2016) Feedback loops of the mammalian circadian clock constitute repressilator. PLoS Comput Biol 12: e1005266. doi:10.1371/journal.pcbi.1005266

14. Thomas R, Thieffry D, Kaufman M (1995) Dynamical behaviour of biological regulatory networks: I. biological role of feedback loops and practical use of the concept of the loop-characteristic state. Bull Math Biol 57: 247-276. doi:10.1016/0092-8240(94)00036-C

15. Kurosawa G, Fujioka A, Koinuma S, Mochizuki A, Shigeyoshi Y (2017) Temperature-amplitude coupling for stable biological rhythms at different temperatures. PLoS Comput Biol 13: e1005501. doi:10.1371/ journal.pcbi.1005501

16. Reppert SM, Weaver DR (2001) Molecular analysis of mammalian circadian rhythms. Annu Rev Physiol 63: 647-676. doi:10.1146/annurev.physiol.63.1.647

17. Relógio A, Westermark PO, Wallach T, Schellenberg K, Kramer A, Herzel H (2011) Tuning the mammalian circadian clock: Robust synergy of two loops. PLoS Comput Biol 7: e1002309. doi:10.1371/journal.pcbi.1002309

18. Stratmann M, Schibler U (2012) REV-ERBs: More than the sum of the individual parts. Cell Metab 15: 791-793. doi:10.1016/j.cmet.2012.05.006

19. Elowitz MB, Leibler S (2000) A synthetic oscillatory network of transcriptional regulators. Nature 403: 335-338. doi:10.1038/35002125

20. Panda S, Antoch MP, Miller BH, Su Al, Schook AB, Straume M, Schultz PG, Kay SA, Takahashi JS, Hogenesch JB (2002) Coordinated transcription of 
key pathways in the mouse by the circadian clock. Cell 109: 307-320. doi:10.1016/s0092-8674(02)00722-5

21. Hughes ME, DiTacchio L, Hayes KR, Vollmers C, Pulivarthy S, Baggs JE, Panda S, Hogenesch JB (2009) Harmonics of circadian gene transcription in mammals. PLoS Genet 5: e1000442. doi:10.1371/journal.pgen.1000442

22. Wang J, Mauvoisin D, Martin E, Atger F, Galindo AN, Dayon L, Sizzano F, Palini A, Kussmann M, Waridel P, et al (2017) Nuclear proteomics uncovers diurnal regulatory landscapes in mouse liver. Cell Metabol 25: 102-117. doi:10.1016/j.cmet.2016.10.003

23. Cho H, Zhao X, Hatori M, Ruth TY, Barish GD, Lam MT, Chong LW, DiTacchio L, Atkins AR, Glass CK, et al (2012) Regulation of circadian behaviour and metabolism by REV-ERB- $\alpha$ and REV-ERB- $\beta$. Nature 485: 123-127. doi:10.1038/nature11048

24. Bugge A, Feng D, Everett LJ, Briggs ER, Mullican SE, Wang F, Jager J, Lazar MA (2012) Rev-erb $\alpha$ and rev-erb $\beta$ coordinately protect the circadian clock and normal metabolic function. Genes Dev 26: 657-667. doi:10.1101/ gad.186858.112

25. Friedel CC, Dölken L, Ruzsics Z, Koszinowski UH, Zimmer R (2009) Conserved principles of mammalian transcriptional regulation revealed by RNA half-life. Nucleic Acids Res 37: e115. doi:10.1093/nar/gkp542

26. Wang J, Symul L, Yeung J, Gobet C, Sobel J, Lück S, Westermark PO, Molina $\mathrm{N}$, Naef F (2018) Circadian clock-dependent and-independent posttranscriptional regulation underlies temporal mrna accumulation in mouse liver. Proc Natl Acad Sci USA 115: E1916-E1925. doi:10.1073/ pnas. 1715225115

27. Schwanhäusser B, Busse D, Li N, Dittmar G, Schuchhardt J, Wolf J, Chen W, Selbach M (2011) Global quantification of mammalian gene expression control. Nature 473: 337-342. doi:10.1038/nature10098

28. Aryal RP, Kwak PB, Tamayo AG, Gebert M, Chiu PL, Walz T, Weitz CJ (2017) Macromolecular assemblies of the mammalian circadian clock. Mol Cell 67: 770-782. doi:10.1016/j.molcel.2017.07.017

29. Masri S, Sassone-Corsi P (2010) Plasticity and specificity of the circadian epigenome. Nature Neurosci 13: 1324-1329. doi:10.1038/nn.2668

30. Chiou YY, Yang Y, Rashid N, Ye R, Selby CP, Sancar A (2016) Mammalian period represses and de-represses transcription by displacing CLOCK-BMAL1 from promoters in a cryptochrome-dependent manner. Proc Natl Acad Sci USA 13: E6072-E6079. doi:10.1073/pnas.1612917113

31. Bintu L, Buchler NE, Garcia HG, Gerland U, Hwa T, Kondev J, Phillips R (2005) Transcriptional regulation by the numbers: Models. Curr Opin Genet Dev 15: 116-124. doi:10.1016/j.gde.2005.02.007

32. Korenčič A, Bordyugov G, Košir R, Rozman D, Goličnik M, Herzel H (2012) The interplay of cis-regulatory elements rules circadian rhythms in mouse liver. PLoS One 7: e46835. doi:10.1371/journal.pone.0046835

33. Rey G, Cesbron F, Rougemont J, Reinke H, Brunner M, Naef F (2011) Genome-wide and phase-specific DNA-binding rhythms of BMAL1 control circadian output functions in mouse liver. PLOS Biol 9:e1000595. doi:10.1371/journal.pbio.1000595

34. Trott AJ, Menet JS (2018) Regulation of circadian clock transcriptional output by CLOCK: BMAL1. PLOS Genet 14: e1007156. doi:10.1371/journal. pgen.1007156

35. Ukai-Tadenuma M, Yamada RG, Xu H, Ripperger JA, Liu AC, Ueda HR (2011) Delay in feed back repression by cryptochrome 1 is required for circadian clock function. Cell 144: 268-281. doi:10.1016/j.cell.2010.12.019

36. Edwards MD, Brancaccio M, Chesham JE, Maywood ES, Hastings MH (2016) Rhythmic expression of cryptochrome induces the circadian clock of arrhythmic suprachiasmatic nuclei through arginine vasopressin signaling. Proc Natl Acad Sci USA 113: 2732-2737. doi:10.1073/pnas.1519044113

37. Vollmers C, Gill S, DiTacchio L, Pulivarthy SR, Le HD, Panda S (2009) Time of feeding and the intrinsic circadian clock drive rhythms in hepatic gene expression. Proc Natl Acad Sci USA 106: 21453-21458. doi:10.1073/ pnas.0909591106
38. Poli R, Kennedy J, Blackwell T (2007) Particle swarm optimization. Swarm Intelligence 1: 33-57. doi:10.1007/s11721-007-0002-0

39. McKay MD, Beckman RJ, Conover WJ (2000) A comparison of three methods for selecting values of input variables in the analysis of output from a computer code. Technometrics 42: 55-61. doi:10.1080/ 00401706.2000.10485979

40. Yamamoto Y, Yagita K, Okamura H (2005) Role of cyclic mPer2 expression in the mammalian cellular clock. Mol Cell Biol 25: 1912-1921. doi:10.1128/ mcb.25.5.1912-1921.2005

41. Numano R, Yamazaki S, Umeda N, Samura T, Sujino M, Takahashi R, Ueda M, Mori A, Yamada K, Sakaki Y, et al (2006) Constitutive expression of the Period1 gene impairs behavioral and molecular circadian rhythms. Proc Natl Acad Sci USA 103: 3716-3721. doi:10.1073/pnas.0600060103

42. Liu AC, Tran HG, Zhang EE, Priest AA, Welsh DK, Kay SA (2008) Redundant function of REV-ERBalpha and beta and non-essential role for Bmal1 cycling in transcriptional regulation of intracellular circadian rhythms. PLoS Genet 4: e1000023. doi:10.1371/journal.pgen.1000023

43. Locke JC, Westermark PO, Kramer A, Herzel H (2008) Global parameter search reveals design principles of the mammalian circadian clock. BMC Systems Biol 2: 22. doi:10.1186/1752-0509-2-22

44. Jolley CC, Ukai-Tadenuma M, Perrin D, Ueda HR (2014) A mammalian circadian clock model incorporating daytime expression elements. Biophys / 107: 1462-1473. doi:10.1016/j.bpj.2014.07.022

45. Izenman AJ (2013) Linear discriminant analysis. In Modern Multivariate Statistical Techniques, pp 237-280. New York, USA: Springer.

46. Kim JK. (2016) Protein sequestration versus Hill-type repression in circadian clock models. IET Systems Biol 10: 125-135. doi:10.1049/ietsyb.2015.0090

47. van der Horst GT, Muijtjens M, Kobayashi K, Takano R, Kanno S, Takao M, de Wit J, Verkerk A, Eker AP, van Leenen D, et al (1999) Mammalian Cry and Cry 2 are essential for maintenance of circadian rhythms. Nature 398: 627-630. doi:10.1038/19323

48. Zheng B, Albrecht U, Kaasik K, Sage M, Lu W, Vaishnav S, Li Q, Sun ZS, Eichele G, Bradley A, et al (2001) Nonredundant roles of the mPer1 and mPer2 genes in the mammalian circadian clock. Cell 105: 683-694. doi:10.1016/s0092-8674(01)00380-4

49. Preitner N, Damiola F, Zakany J, Duboule D, Albrecht U, Schibler U (2002) The orphan nuclear receptor REV-ERBa controls circadian transcription within the positive limb of the mammalian circadian oscillator. Cell 110: 251-260. doi:10.1016/s0092-8674(02)00825-5

50. Yan J, Wang H, Liu Y, Shao C (2008) Analysis of gene regulatory networks in the mammalian circadian rhythm. PLoS Comput Biol 4: e1000193. doi:10.1371/journal.pcbi.1000193

51. Bozek K, Relógio A, Kielbasa SM, Heine M, Dame C, Kramer A, Herzel H (2009) Regulation of clock-controlled genes in mammals. PLoS One 4 e4882. doi:10.1371/journal.pone.0004882

52. Yang X, Downes M, Ruth TY, Bookout AL, He W, Straume M, Mangelsdorf DJ, Evans RM (2006) Nuclear receptor expression links the circadian clock to metabolism. Cell 126: 801-810. doi:10.1016/j.cell.2006.06.050

53. Bass J, Takahashi JS (2010) Circadian integration of metabolism and energetics. Science 330: 1349-1354. doi:10.1126/science.1195027

54. Mukherji A, Kobiita A, Damara M, Misra N, Meziane H, Champy MF, Chambon P (2015) Shifting eating to the circadian rest phase misaligns the peripheral clocks with the master SCN clock and leads to a metabolic syndrome. Proc Natl Acad Sci USA 112: E6691-E6698. doi:10.1073/pnas.1519735112

55. Myung J, Hong S, Hatanaka F, Nakajima Y, De Schutter E, Takumi T (2012) Period coding of Bmal1 oscillators in the suprachiasmatic nucleus. J Neurosci 32: 8900-8918. doi:10.1523/jneurosci.5586-11.2012

56. Ono D, Honma S, Nakajima Y, Kuroda S, Enoki R, Honma KI (2017) Dissociation of Per1 and Bmal1 circadian rhythms in the 
suprachiasmatic nucleus in parallel with behavioral outputs. Proc Natl Acad Sci USA 114: E3699-E3708. doi:10.1073/pnas.1613374114

57. Tokuoka A, Itoh TQ, Hori S, Uryu O, Danbara Y, Nose M, Bando T, Tanimura T, Tomioka K (2017) Cryptochrome genes form an oscillatory loop independent of the per/tim loop in the circadian clockwork of the cricket Gryllus bimaculatus. Zoological Lett 3: 5. doi:10.1186/s40851-017-0066-7

58. Aton SJ, Colwell CS, Harmar AJ, Waschek J, Herzog ED (2005) Vasoactive intestinal polypeptide mediates circadian rhythmicity and synchrony in mammalian clock neurons. Nat Neurosci 8: 476-483. doi:10.1038/nn1419

59. Gonze D, Bernard S, Waltermann C, Kramer A, Herzel H (2005) Spontaneous synchronization of coupled circadian oscillators. Biophys J 89: 120-129. doi:10.1529/biophysj.104.058388

60. Granada AE, Bordyugov G, Kramer A, Herzel H (2013) Human chronotypes from a theoretical perspective. PLoS One 8: e59464. doi:10.1371/journal. pone.0059464
61. Farajnia S, van Westering TL, Meijer JH, Michel S (2014) Seasonal induction of GABAergic excitation in the central mammalian clock. Proc Natl Acad Sci USA 111: 9627-9632. doi:10.1073/pnas.1319820111

62. Azzi A, Evans JA, Leise T, Myung J, Takumi T, Davidson AJ, Brown SA. (2017) Network dynamics mediate circadian clock plasticity. Neuron 93: 441-450. doi:10.1016/j.neuron.2016.12.022

63. Woller A, Duez H, Staels B, Lefranc M (2016) A mathematical model of the liver circadian clock linking feeding and fasting cycles to clock function. Cell Rep 17: 1087-1097. doi:10.1016/j.celrep.2016.09.060

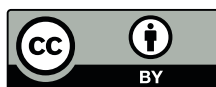

License: This article is available under a Creative Commons License (Attribution 4.0 International, as described at https://creativecommons.org/ licenses/by/4.0/). 